\title{
Critical role of deoxynucleotidyl transferase terminal interacting protein 1 in oral cancer
}

\author{
Yuki Sawai $^{1}$ - Atsushi Kasamatsu ${ }^{2} \cdot$ Dai Nakashima $^{2} \cdot$ Kazuaki Fushimi $^{3} \cdot$ Hiroki Kasama $^{3} \cdot$ Manabu lyoda $^{4}$. \\ Yukinao Kouzu $^{5} \cdot$ Masashi Shiiba $^{6} \cdot$ Hideki Tanzawa $^{1,2} \cdot$ Katsuhiro Uzawa ${ }^{1,2}$
}

Received: 9 December 2017 / Revised: 19 March 2018 / Accepted: 3 April 2018 / Published online: 31 May 2018

(c) United States \& Canadian Academy of Pathology 2018

\begin{abstract}
Deoxynucleotidyl transferase terminal interacting protein 1 (DNTTIP1) forms a complex with histone deacetylase (HDAC); however, the relevance of DNTTIP1 in cancer remains unknown. The aim of this study was to examine DNTTIP1 expression and its functional mechanisms in oral squamous cell carcinomas (OSCCs). DNTTIP1 expression was analyzed by quantitative reverse transcriptase-polymerase chain reaction, immunoblotting analysis, and immunohistochemistry. The expression of DNTTIP1 was upregulated significantly in vitro and in vivo, and in patients with OSCC in whom DNTTIP1 was overexpressed and the expression level was correlated significantly $(P<0.05)$ with tumoral growth. DNTTIP1 knockdown (siDNTTIP1) cells showed depressed cellular proliferation by cell-cycle arrest at the G1 phase with high acetylation of $\mathrm{p} 53$ and upregulation of $\mathrm{p} 21^{\mathrm{Cip} 1}$. Moreover, resveratrol, a HDAC inhibitor, controlled not only acetylated p53 status but also DNTTIP1 expression, leading to a similar phenotype of siDNTTIP1 cells. A marked $(P<0.05)$ reduction of tumoral growth in mouse xenograft models was observed with lower DNTTIP1 expression under the presence of this chemical reagent. Taken together, our results suggested that DNTTIP1-HDAC interaction promotes tumoral growth through deacetylation of p53 and that DNTTIP1 might be a critical therapeutic target in OSCCs.
\end{abstract}

\section{Introduction}

Deoxynucleotidyl transferase terminal interacting protein 1 (DNTTIP1) was identified originally as a binding partner of DNA polymerase [1]. The detailed functions of DNTTIP1 have not been clarified yet; however, high expression has

$\triangle$ Atsushi Kasamatsu

kasamatsua@faculty.chiba-u.jp

$\triangle$ Katsuhiro Uzawa

uzawak@faculty.chiba-u.jp

1 Department of Oral Science, Graduate School of Medicine, Chiba University, Chiba, Japan

2 Department of Dentistry and Oral-Maxillofacial Surgery, Chiba University Hospital, Chiba, Japan

3 Department of Dentistry and Oral Surgery, Eastern Chiba Medical Center, Chiba, Japan

4 Division of Oral Surgery, Chiba Rosai Hospital, Chiba, Japan

5 Division of Dentistry, Chiba Children's Hospital, Chiba, Japan

6 Department of Medical Oncology, Graduate School of Medicine, Chiba University, Chiba, Japan been reported in muscles [2]. To date, no reports regarding DNTTIP1 related to cancer research have been published.

DNTTIP has two isoforms, DNTTIP1 and DNTTIP2 [3]. The former enhances the DNA polymerase activity and the latter suppresses its activity [4]. Our microarray analysis showed that DNTTIP1 was upregulated in oral squamous cell carcinomas (OSCC) cells compared with human normal oral keratinocytes:HNOKs (cultured from the oral mucosal epithelium of young healthy patients) [5].

Epigenetic abnormalities are believed to be involved in all stages of cancer progression [6]. Histone acetylation is one of the most investigated pathways for epigenetic regulation [6]. Histone deacetylases (HDACs) are associated closely with the development and progression of cancer and have been reported to cause deacetylation of p53 [7]. p53 deacetylation is assumed to be associated with loss of cancer-suppressing ability, and induction of apoptosis of cancer cells is less likely to occur [8]. HDAC1 forms a complex with DNTTIP1 [9, 10]. Therefore, here, we assumed that DNTTIP1 plays some roles in deacetylation of p53 by HDAC1.

In this study, DNTTIP1 was overexpressed in OSCCderived cell lines and primary OSCCs, and, therefore, we 
hypothesized that DNTTIP1 might be associated with OSCC. We also clarified the clinical relevance of DNTTIP1 in cancer using a knockdown model and resveratrol, an HDAC inhibitor.

\section{Materials and methods}

\section{Ethics statement}

The ethical committee of the Graduate School of Medicine, Chiba University, approved the research protocol (protocol number 680). All patients provided written informed consent before inclusion in the study.

\section{Cells and tissue samples}

Human OSCC-derived cell lines were purchased from RIKEN BioResource Center (Tsukuba, Ibaraki, Japan) and the Japanese Collection of Research Bioresources Cell Bank (Ibaraki, Osaka, Japan) [11-13]. We obtained HNOKs from young healthy patients and cultured them as described previously [14-17]. Three independent HNOKs were primarily cultured and passaged five times in oral keratinocyte medium (ScienCell Research Laboratories, Carlsbad, CA) and $5 \mathrm{ml}$ of penicillin/streptomycin solution (ScienCell Research Laboratories). The HNOKs served as normal control cells.

\section{mRNA expression analysis}

Quantitative reverse transcriptase-PCR (qRT-PCR) was carried out as described previously [18-22]. The sequences of the gene-specific primers and universal probes were as follows: DNTTIP1 (5'-CCTCGATGAGGAGGTAGGC-3' and 5'-CAGCTGACCCCCAGGATA-3') and universal probe \#20, and glyceraldehyde-3-phosphate dehydrogenase (GAPDH) (5'-AGCCACATCGCTCAGACAC-3' and 5'GCCCAATACGACCAAATCC-3') and universal probe \#60.

\section{Immunoblotting analysis}

Protein extraction and immunoblotting analysis were conducted as described previously [23-26]. Protein concentrations were determined using the Bradford concentration assay (Bradford reagent) and a TECAN Infinite 200 PRO (Tecan Group Ltd., Mannedorf, Switzerland) microplate reader and unified to $20 \mu \mathrm{g}$ per lane. The antibodies were anti-DNTTIP1 (sc-98407, rabbit), antiGAPDH (sc-25778, rabbit), anti-cyclin E (sc-377100, mouse) (Santa Cruz Biotechnology; Santa Cruz, CA, USA), anti-CDK2 (\#2546, rabbit), anti-CDK4 (\#12790, rabbit),
anti-CDK6 (\#3136, mouse), anti-p21 ${ }^{\text {Cip1 }}$ (\#2947, rabbit), anti-p27 ${ }^{\text {Kip1 }}$ (\#3686, rabbit), anti-cyclin D1 (\#2978, rabbit), anti-p53 (\#2524, rabbit), anti-acetyl-p53 (\#2525), and antiHDAC1 (\#34589) (Cell Signaling Technology, Danvers, MA, USA).

\section{Immunohistochemistry (IHC)}

IHC was carried out as described previously [27-31]. The DNTTIP1 IHC score was calculated by combining the proportion and intensity of DNTTIP1 stained cells. The intensities of the stained cells were classified as 0 , no staining; 1 , weak staining; 2, moderate staining; and 3, strong staining.

\section{Transfection with siRNA plasmid}

Transfection with siRNA was conducted as described previously $[18,19]$. DNTTIP1 siRNA (siDNTTIP1) and control siRNA (siControl) (sc-76640, sc-37007, Santa Cruz Biotechnology) were transfected into $\mathrm{HSC} 2$ and $\mathrm{HSC} 4$ cells. To investigate the efficiency of DNTTIP1 knockdown, we carried out qRT-PCR and immunoblotting analysis.

\section{Cellular proliferation assay}

To examine the effects of DNTTIP1 knockdown and resveratrol treatment on cellular growth, we performed the cellular proliferation assay as described previously [23-26].

\section{Cell-cycle analysis}

The transfectants were treated with $200 \mathrm{ng} / \mathrm{ml}$ nocodazole (Sigma-Aldrich, St. Louis, MO, USA) for $16 \mathrm{~h}$ to synchronize cells at the G2/M transition [32, 33]. After treatment, the cells were collected, washed with phosphatebuffered saline, and processed with the CycleTEST Plus DNA reagent kit (Becton-Dickinson, Franklin Lakes, NJ, USA). Flow cytometric determination of the DNA content was analyzed using the BD AccuriTM C6 Flow Cytometer (Becton-Dickinson) [23-26].

\section{Resveratrol treatment}

Resveratrol (Sigma-Aldrich), a natural phytochemical in fruits, enhances p53 acetylation and suppresses the HDAC1 activity in several types of cancers [31-34]. To investigate whether resveratrol treatment was related to cellular proliferation and cell cycle, the cells were subjected to treatment with resveratrol $(50 \mu \mathrm{M})$ for $48 \mathrm{~h}$. We used dimethylsulfoxide (DMSO) as excipients in vitro and in vivo. 


\section{In vivo tumor xenograft study}

To investigate the cellular proliferation inhibitory effect of resveratrol, we used xenograft models (HSC-2 and HSC-4). The cells $\left(1 \times 10^{7}\right.$ cells $)$ were injected independently and subcutaneously. Female nude mice (6-week-old, BALB/cA$\mathrm{nu}[\mathrm{nu} / \mathrm{nu}])$ were purchased from Oriental Yeast Co. (Tokyo, Japan). The volume of the implanted tumor into the dorsal side of the mice was measured with a digital caliper every 3-4 days after injection. We used the formula $4 \pi / 3 \times$ (width/2) $)^{2} \times($ length/2) to calculate tumoral volume. After the tumors reached approximately $100 \mathrm{~mm}^{3}$ ( 10 days), daily resveratrol intraperitoneal injections $(2 \mathrm{mg}$ of resveratrol in $50 \mu$ l of DMSO) started for 28 days (three mice/ group), at which time the mice were sacrificed, and tumoral tissues were collected for further analyses.

\section{Statistical analysis}

Statistical differences were analyzed by the $\chi^{2}$ test, Student's $t$-test, Fisher's exact test, and Mann-Whitney U-test. $P<0.05$ indicates statistically significant. All data are expressed as the mean \pm standard error (SEM).

\section{Results}

\section{Upregulation of DNTTIP1 in OSCC cells}

To evaluate the status of DNTTIP1 expression, we conducted qRT-PCR and performed immunoblotting analysis using the nine OSCC cell lines and HNOKs, as normal control cells. DNTTIP1 mRNA expression was upregulated significantly in all nine OSCC cell lines compared with the HNOKs (Fig. 1a, $P<0.05$ ). Figure $1 \mathrm{~b}$ shows representative results of immunoblotting analysis. The DNTTIP1 protein also increased in all nine OSCC cell lines compared with their counterparts.

\section{Evaluation of DNTTIP1 expression in primary OSCC samples and clinical correlations of clinicopathological features}

We evaluated the DNTTIP1 expression in primary OSCCs by IHC. High DNTTIP1 expression was found in the nuclei of the OSCC specimens; the normal oral tissues showed almost negative immunostaining (Fig. 2a). Representative IHC data for DNTTIP1 in normal tissue and primary OSCC are shown in Fig. 2a. The scores in the normal oral tissues ranged from 2.5 to 75 (median, 33.05) and in primary OSCC tissues ranged from 18.8 to 195.6 (median, 104.5). These IHC scores in primary OSCCs were clearly $(P<0.05)$ greater than in normal oral tissues (Fig. 2b). The
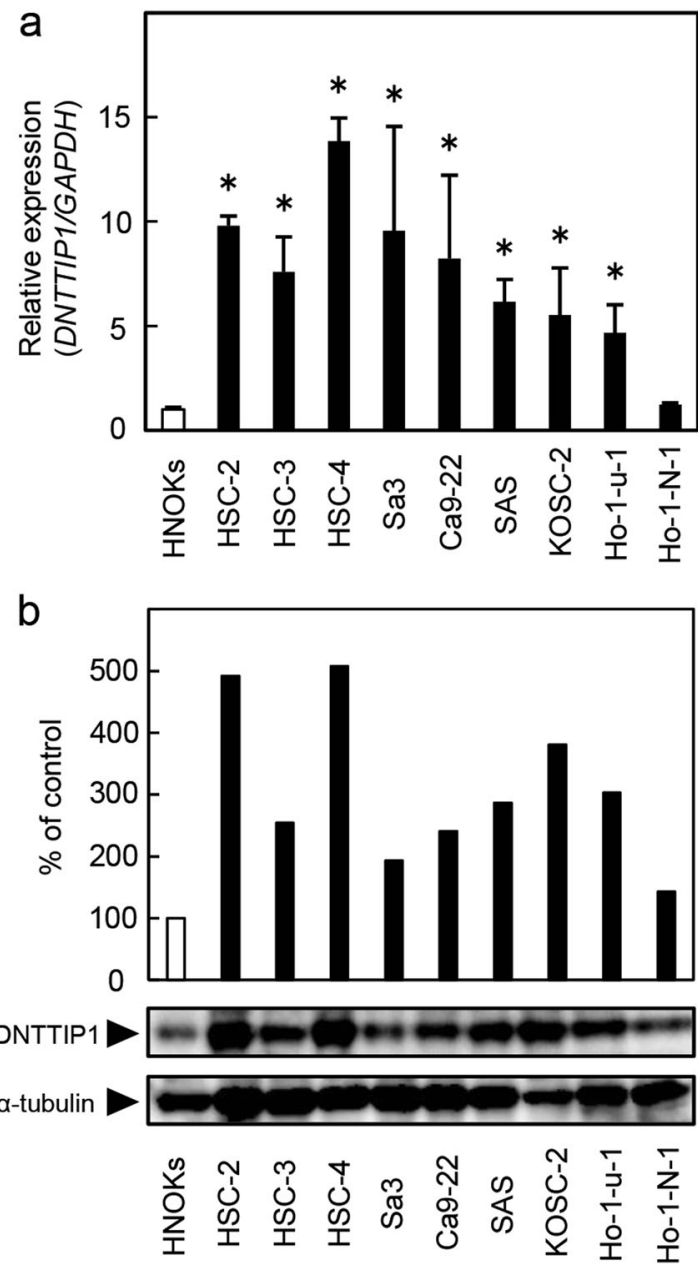

Fig. 1 Upregulation of DNTTIP1 expression in OSCC cells. a Quantification of DNTTIP1 mRNA expression in OSCC cells by qRT-PCR analysis. Significant $(* P<0.05$, Student's $t$-test) upregulation of DNTTIP1 mRNA is seen in nine OSCC cells compared with the HNOKs. The data are expressed as the mean \pm SEM of three outcomes. b Immunoblotting analysis of DNTTIP1 protein in OSCC cells and HNOKs. The expression of DNTTIP1 protein is upregulated in OSCC cells compared with the HNOKs. The densitometric DNTTIP1 protein data are normalized to the GAPDH protein levels. The values are expressed as the percentage of the HNOKs

correlations between the clinicopathological features of patients with OSCC and the state of DNTTP1 protein expression are shown in Table 1. Of the clinical parameters, the DNTTIP1 expression level was clearly $(P<0.05)$ related to the primary tumoral size of the OSCCs.

\section{siRNA knockdown of DNTTIP1 from OSCC cells}

Since overexpression of DNTTIP1 was seen in OSCC in vitro and in vivo (Figs. 1 and 2), we transiently transfected siDNTTIP1 and siControl into OSCC cells (HSC-2 and HSC-4). To investigate the efficiency of the transfection, we performed qRT-PCR and immunoblotting analysis. DNTTIP1 mRNA expression in the siDNTTIP1 cells was 

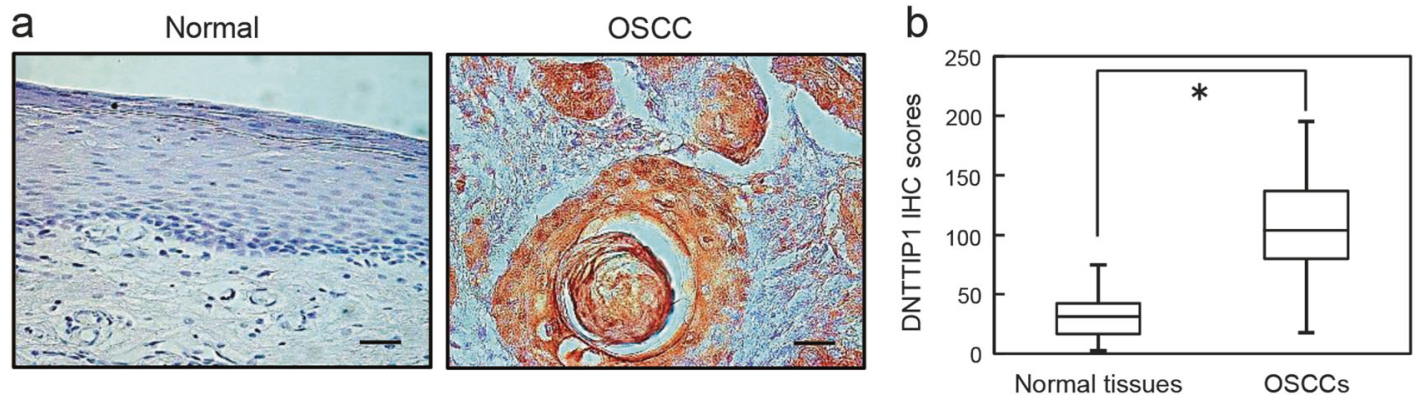

Fig. 2 Evaluation of DNTTIP1 expression in primary OSCC samples. a Representative IHC results of DNTTIP1 protein in normal oral tissue and primary OSCCs (original magnification: 400×, Scale bars: $50 \mu \mathrm{m}$ ). b The status of DNTTIP1 protein expression in normal counterparts based on the primary OSCC $(n=105)$ and IHC scoring system. The

clearly $(P<0.05)$ lower than that in the siControl cells (Fig. 3a). DNTTIP1 protein levels in the siDNTTIP1 cells also decreased compared with the siControl cells (Fig. 3b).

\section{Cellular growth and cell-cycle analysis of siDNTTIP1 cells}

To evaluate the effect of DNTTIP1 knockdown on cellular growth, a cellular proliferation assay was performed. We found a significant $(P<0.05)$ decrease in cellular proliferation in the siDNTTIP1 cells compared with the siControl cells (Fig. 4a). We also assessed the cell-cycle distribution by flow cytometry (Fig. 4b), since the cellular growth of the DNTTIP1 knockdown cells decreased compared with the control. The percentage of the siDNTTIP1 cells at the G1 phase was clearly $(P<0.05)$ higher than that of the siControl cells (Fig. 4b). Assessment of the expression levels of the cyclin-dependent kinase inhibitors (CDKIs) (p21 $1^{\mathrm{Cip} 1}$ and p27 $7^{\mathrm{Kip} 1}$ ), CDKs (CDK2, CDK4, and CDK6), and cyclins (cyclin D1 and cyclin E) showed that the CDKIs were upregulated, and CDKs and cyclins were downregulated in the siDNTTIP1 cells (Fig. 4c). These results indicated that siDNTTIP1 inhibited cellular proliferation by cell-cycle arrest at the G1 phase.

\section{Resveratrol treatment}

To investigate the effect of resveratrol, we first performed the cellular proliferation assay. The cellular growth of the resveratrol-treated cells was significantly $(P<0.05)$ lower than that of control cells (HSC-2 and HSC-4) (Fig. 5a). In the cell-cycle analysis, the percentage of the resveratroltreated cells at the G1 phase was clearly $(P<0.05)$ higher than that of control cells (Fig. 5b). Assessment of the expression levels of DNTTIP1, CDKIs, CDKs, and cyclins using immunoblotting analysis after treatment with resveratrol showed that the DNTTIP1, CDKs, and cyclin levels were downregulated, and the CDKI levels were upregulated
DNTTIP1 IHC scores in normal oral tissues range from 2.5 to 75 (median: 33.05) and in primary OSCC tissues from 18.8 to 195.6 (median:104.5). The DNTTIP1 protein expression levels in OSCCs are markedly $(* P<0.05$, Student's $t$-test) higher than in the normal oral tissues

in the resveratrol-treated cells compared with the control cells (Fig. 5c).

\section{p53, acetyl-p53, and HDAC1 expression levels in DNTTIP1 knockdown cells and resveratrol-treated cells}

To investigate the effect of DNTTIP1 knockdown and resveratrol treatment, we assessed p53, acetyl-p53, and HDAC1 expression levels using immunoblotting analysis. The acetyl-p53 levels were upregulated in the siDNTTIP1 and resveratrol-treated cells. The p53 and HDAC1 levels did not change after siRNA and resveratrol treatments (Fig. 6).

\section{Resveratrol inhibited tumoral growth in vivo}

We appraised the effect of resveratrol on tumoral growth in vivo using target tumor xenografts in nude mice. OSCC cells (HSC-2 and HSC-4) were injected subcutaneously into the backs of female nude mice (three mice in each group). The tumoral volume of the resveratrol-treated group was clearly $(P<0.05)$ smaller than that of the control group (Fig. 7a). After resveratrol treatment, the xenografted tumors showed a significant decrease in DNTTIP1 expression (Fig. 7b). Resveratrol did not affect the body weight of the mice compared with the control group (data not shown).

\section{Discussion}

In the current study, overexpressed DNTTIP1 was observed in OSCC in vitro and in vivo and was correlated positively with tumoral growth. The siDNTTIP1 cells showed decreased cellular proliferation through cell-cycle arrest at the G1 phase. Interestingly, resveratrol, the HDAC inhibitor, controlled DNTTIP1 expression and led to a similar phenotype of DNTTIP1 knockdown cells [35-37]. 
Table 1 Correlation between DNTTIP1 expression and clinical classification in OSCCs

\begin{tabular}{|c|c|c|c|c|}
\hline \multirow{3}{*}{$\begin{array}{l}\text { Clinical } \\
\text { classification }\end{array}$} & \multirow[b]{3}{*}{ Total } & \multicolumn{2}{|c|}{ Results of immunostaining } & \multirow[b]{3}{*}{$\mathrm{P}$ value } \\
\hline & & \multicolumn{2}{|c|}{ No. of patients } & \\
\hline & & $\begin{array}{l}\text { DNTTIP1 } \\
\text { negative }\end{array}$ & $\begin{array}{l}\text { DNTTIP1 } \\
\text { positive }\end{array}$ & \\
\hline \multicolumn{5}{|c|}{ Age at surgery (years) } \\
\hline$<70$ & 60 & 27 & 33 & \multirow[t]{2}{*}{$0.26^{\mathrm{a}}$} \\
\hline$\geqq 70$ & 45 & 23 & 22 & \\
\hline \multicolumn{5}{|l|}{ Gender } \\
\hline Male & 57 & 27 & 30 & \multirow[t]{2}{*}{$0.48^{\mathrm{b}}$} \\
\hline Female & 48 & 23 & 25 & \\
\hline \multicolumn{5}{|c|}{ T-primary tumor } \\
\hline $\mathrm{T} 1$ & 27 & 15 & 12 & \\
\hline $\mathrm{T} 2$ & 33 & 15 & 18 & \\
\hline $\mathrm{T} 3$ & 8 & 3 & 5 & \\
\hline $\mathrm{T} 4$ & 37 & 10 & 27 & \\
\hline $\mathrm{T} 1+\mathrm{T} 2$ & 60 & 30 & 30 & \multirow[t]{2}{*}{$0.032^{b^{*}}$} \\
\hline $\mathrm{T} 3+\mathrm{T} 4$ & 45 & 13 & 32 & \\
\hline \multicolumn{5}{|c|}{ N-regional lymph node } \\
\hline Negative & 65 & 28 & 37 & \multirow[t]{2}{*}{$0.07^{\mathrm{b}}$} \\
\hline Positive & 40 & 16 & 24 & \\
\hline \multicolumn{5}{|l|}{ TNM stage } \\
\hline I + II & 46 & 20 & 26 & \multirow[t]{2}{*}{$0.064^{\mathrm{b}}$} \\
\hline III + IV & 59 & 23 & 36 & \\
\hline \multicolumn{5}{|c|}{ Vascular invasion } \\
\hline Negative & 70 & 30 & 40 & \multirow[t]{2}{*}{$0.081^{\mathrm{b}}$} \\
\hline Positive & 35 & 13 & 22 & \\
\hline \multicolumn{5}{|c|}{ Histopathologic type } \\
\hline $\begin{array}{l}\text { Well } \\
\text { moderately }\end{array}$ & 99 & 42 & 57 & \multirow[t]{2}{*}{$0.38^{\mathrm{c}}$} \\
\hline Poorly & 6 & 1 & 5 & \\
\hline \multicolumn{5}{|l|}{ Tumoral site } \\
\hline Tongue & 51 & 11 & 40 & \multirow[t]{4}{*}{$0.56^{\mathrm{c}}$} \\
\hline Gingiva & 33 & 7 & 26 & \\
\hline Oral floor & 7 & 1 & 6 & \\
\hline $\begin{array}{l}\text { Buccal } \\
\text { mucosa }\end{array}$ & 14 & 3 & 11 & \\
\hline
\end{tabular}

*Indicates significance

${ }^{\mathrm{a}} \chi^{2}$ test

${ }^{\mathrm{b}}$ Fisher's exact test

${ }^{\mathrm{c}}$ Mann-Whitney U-test

A recent study reported that DNTTIP1 binds to HDAC1 and forms a complex [9, 10, 38]. Although the detailed functions of DNTTIP1 in cancer remain unknown, HDAC1, the binding partner of DNTTIP1, is associated closely with the development and progression of cancers [7]. HDACs
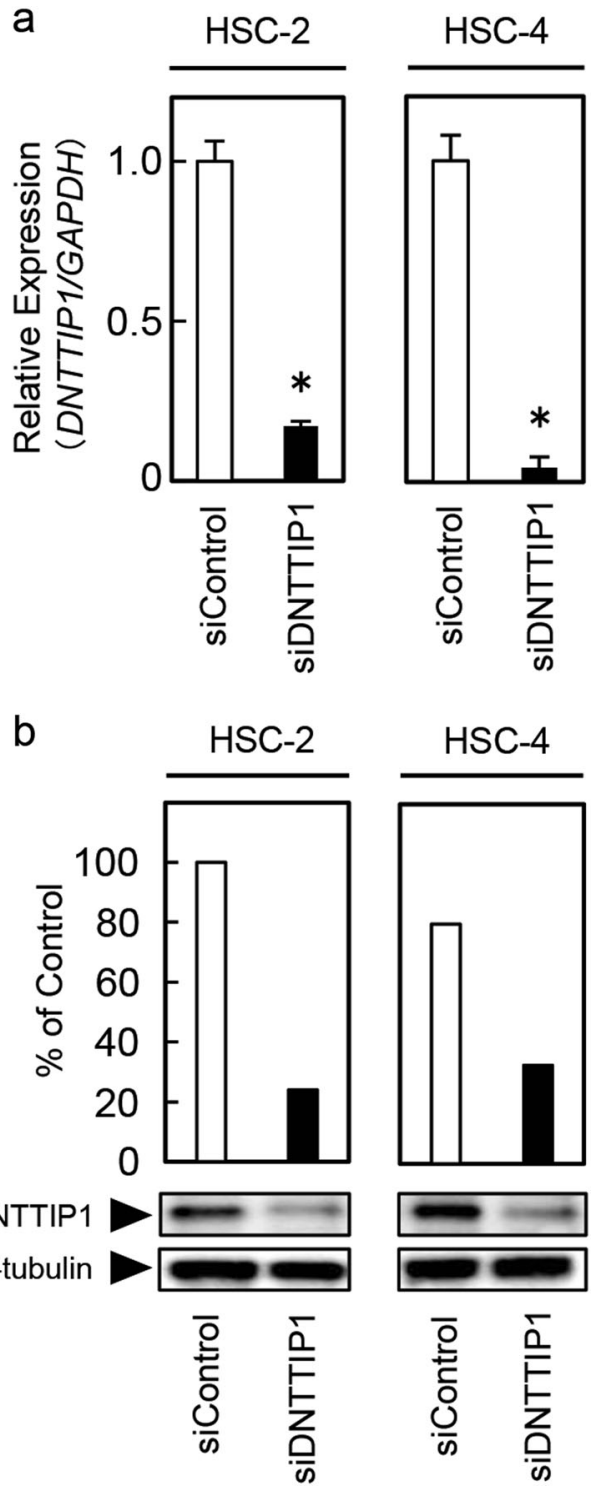

Fig. 3 siRNA knockdown of DNTTIP1 from OSCC cells. a Expression of DNTTIP1 mRNA in the siDNTTIP1 cells (HSC-2 and HSC-4derived transfectants). DNTTIP1 mRNA expression in the siDNTTIP1 cells is clearly $\left({ }^{*} P<0.05\right.$, Student's $t$-test) downregulated compared with the siControl cells. b Immunoblotting analysis shows that the DNTTIP1 protein levels in the siDNTTIP1 cells are also markedly lower compared with the siControl cells

cause deacetylation of p53 [8], leading to loss of the tumoral suppressing function of p53. Acetylated p53 upregulates $\mathrm{p} 21^{\text {Cip } 1}$ to direct cell-cycle arrest at the G1 phase [39-41]. Similar to previous data, our DNTTIP1 knockdown cells also showed cell-cycle arrest at the G1 phase with upregulation of $\mathrm{p} 21^{\mathrm{Cip} 1}$ and $\mathrm{p} 27^{\mathrm{Kip} 1}$ and downregulation of cyclin D1 and cyclin E. Members of the Cip/Kip family are implicated in negative regulation of cell-cycle progression from the $\mathrm{G} 1$ to $\mathrm{S}$ phase by binding and modulating CDK [39-41]. 

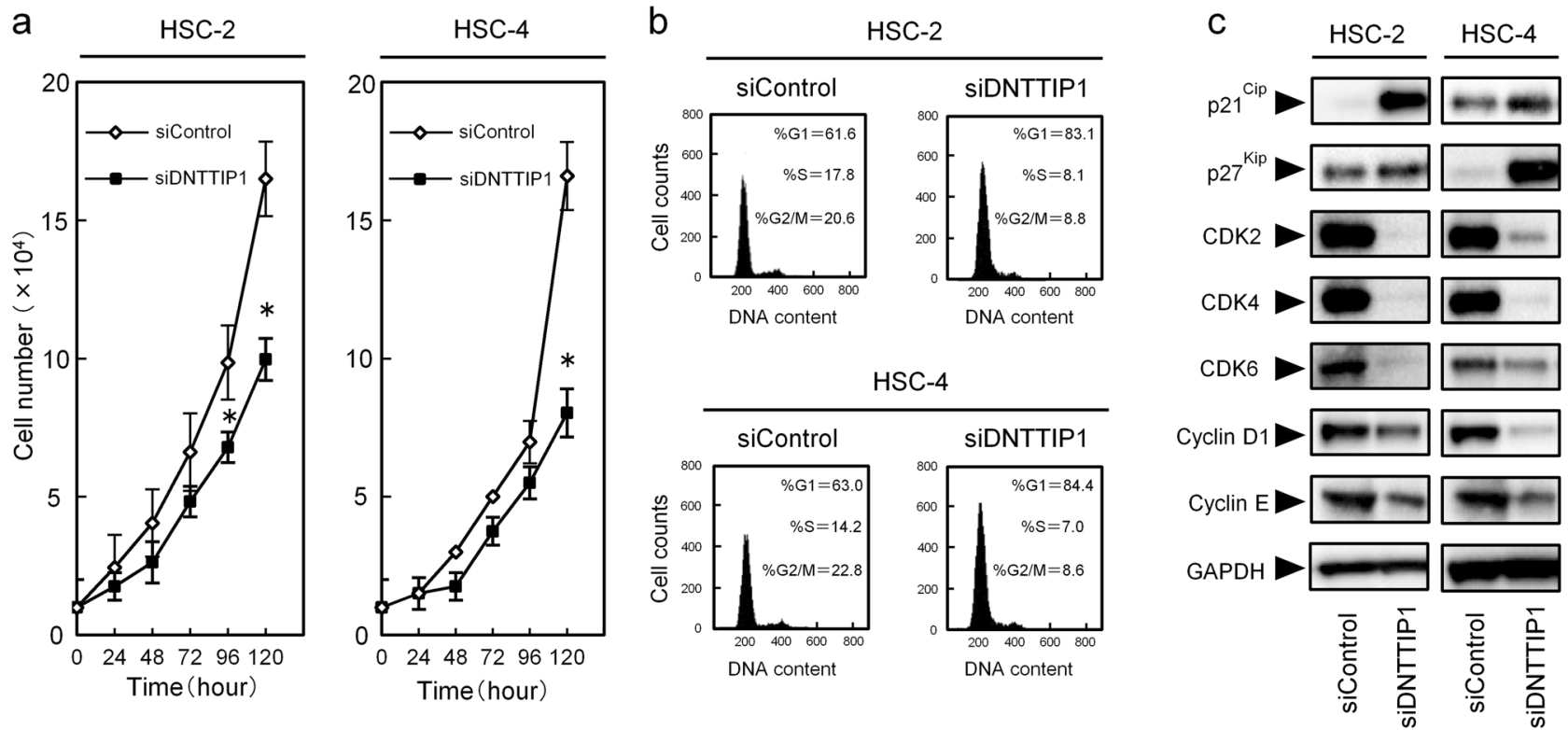

Fig. 4 Cellular growth and cell-cycle analysis of siDNTTIP1 cells. a To determine the effect of knockdown of DNTTIP1 on cellular proliferation, the siDNTTIP1 and siControl cells were seeded in $6-\mathrm{cm}$ dishes at a density of $1 \times 10^{4}$ viable cells. Both transfectants were counted on 5 consecutive days. The cellular growth of the siDNTTIP1 cells is inhibited significantly compared with the siControl cells after 5 days $(120 \mathrm{~h})$. The results are expressed as the means \pm SEM of values from three assays $(* P<0.05$, Student's $t$-test). b A flow cytometric analysis is performed to investigate cell-cycle progression in the siDNTTIP1 and siControl cells after synchronization at the $\mathrm{G} 2 / \mathrm{M}$ phase using nocodazole. The percentage of the siDNTTIP1 cells in the G1 phase is increased $(P<0.05$, Mann-Whitney U-test) compared with the siControl cells. c Immunoblotting analysis shows upregulation of $\mathrm{p} 21^{\mathrm{Cip} 1}$ and $\mathrm{p} 27^{\mathrm{Kip} 1}$ and downregulation of CDK2, CDK4, CDK6, cyclin D1, and cyclin E in the siDNTTIP1 cells compared with the siControl cells a

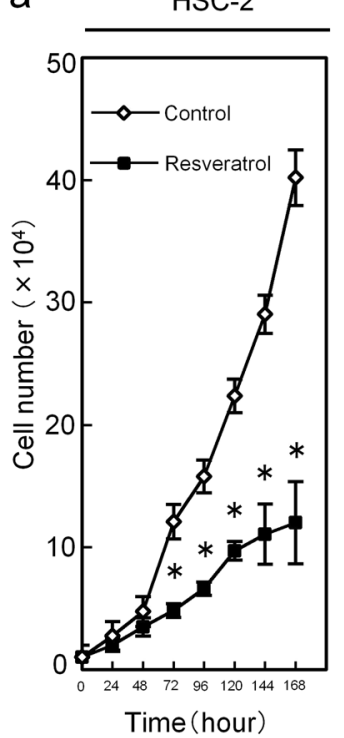

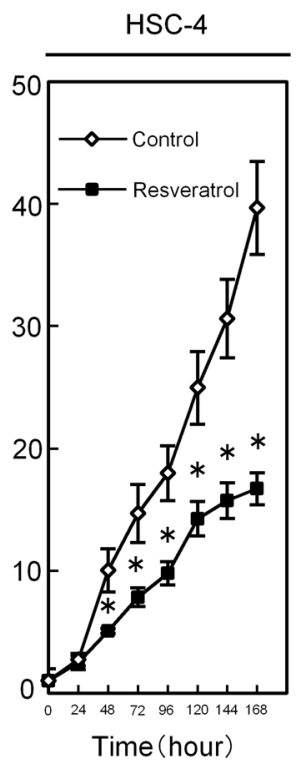

b
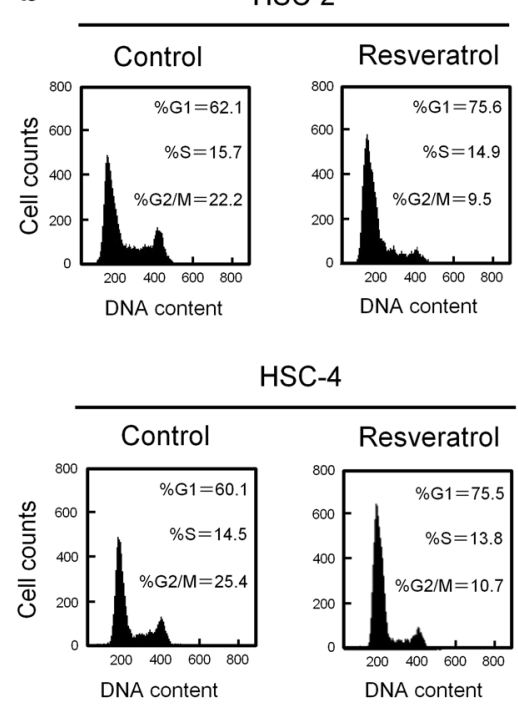

C

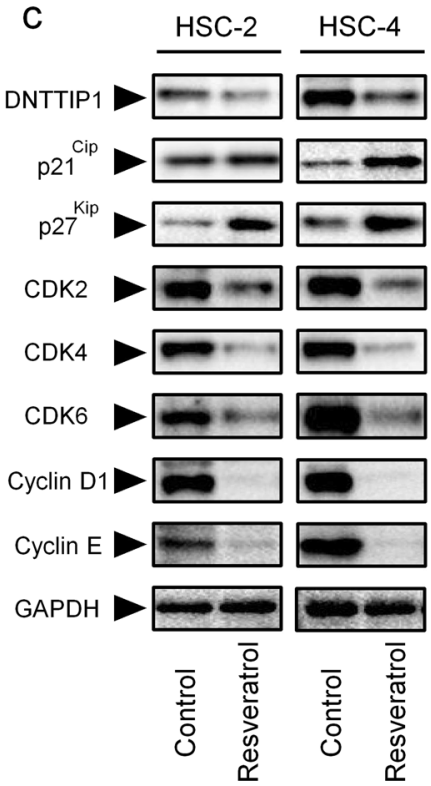

Fig. 5 Resveratrol treatment. a To assess the effect of resveratrol on cellular proliferation, the resveratrol-treated cells and control cells were seeded on culture plates. Both transfectants were counted on 7 consecutive days. The cellular growth of the resveratrol-treated cells is significantly lower than that of the control cells after 7 days $(168 \mathrm{~h})$. The results are expressed as the means \pm SEM of values from three assays $(* P<0.05$, Student's $t$-test). b A flow cytometric analysis is performed to investigate cell-cycle progression in the control cells (HSC-2 and HSC-4) and resveratrol-treated cells using nocodazole. The percentage of resveratrol-treated cells in the G1 phase is increased $(P<0.05$, Mann-Whitney U-test $)$ compared with the control cells. c Immunoblotting analysis shows downregulation of DNTTIP1, CDKs, and cyclins and upregulation of CDKIs in the resveratrol-treated cells compared with the control cells 


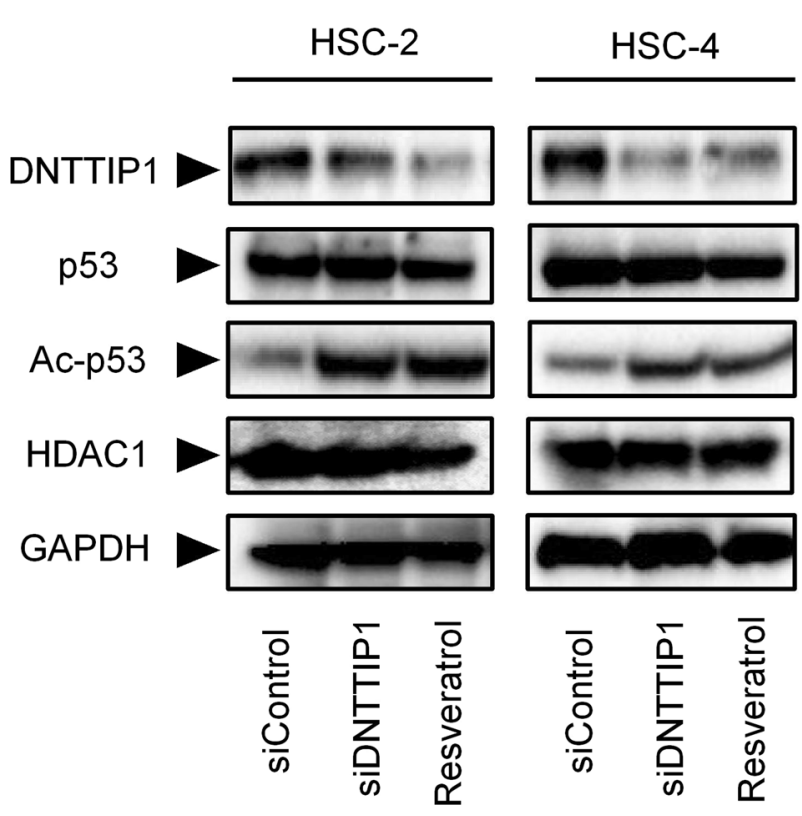

Fig. 6 p53, acetyl-p53, and HDAC1 expression levels in the DNTTIP1 knockdown and resveratrol-treated cells. Immunoblotting analysis shows upregulation of acetyl-p53 levels in the siDNTTIP1 and resveratrol-treated cells compared with the control cells. The p53 and HDAC1 levels did not change after siRNA and resveratrol treatments

Resveratrol modulates multiple cellular signaling pathways, such as the antioxidant, anti-inflammatory, and inhibitory activities of tumoral growth [42-49]. In silico docking analysis showed that resveratrol has the highest binding capacity of all the 11 human HDAC enzymes [50, 51]. Resveratrol promotes acetylation of p53 and thus increases the expression of p53-target genes that participate in cell-cycle arrest $[52,53]$.

Since siDNTTIP1 inhibited HDAC1 activity leading to increased acetylated p53, we investigated whether resveratrol regulated not only HDAC activity but also DNTTIP1 expression. In addition to the in vitro experiments using resveratrol, xenografted tumors after intraperitoneal injection with resveratrol were characterized by inhibited tumoral growth and negative staining for DNTTIP1 by IHC (Fig. 7b), indicating that DNTTIP1 silencing and resveratrol treatment have similar effects on cancer progression. Although resveratrol is compatible with the binding pocket of several HDACs as the inhibitor, DNTTIP1 interacts with the specific target, HDAC1. Therefore, the non-targeting effects of DNTTIP1 are substantially lower than those of resveratrol.

In conclusion, this study is the first to reveal that DNTTIP1 plays a crucial role in cancer progression in OSCC by controlling the status of p53 acetylation through DNTTIP1-HDAC1 interaction. Thus, our data suggested that DNTTIP1 might be a potential biomarker for cancer growth and a novel therapeutic target in OSCCs. a
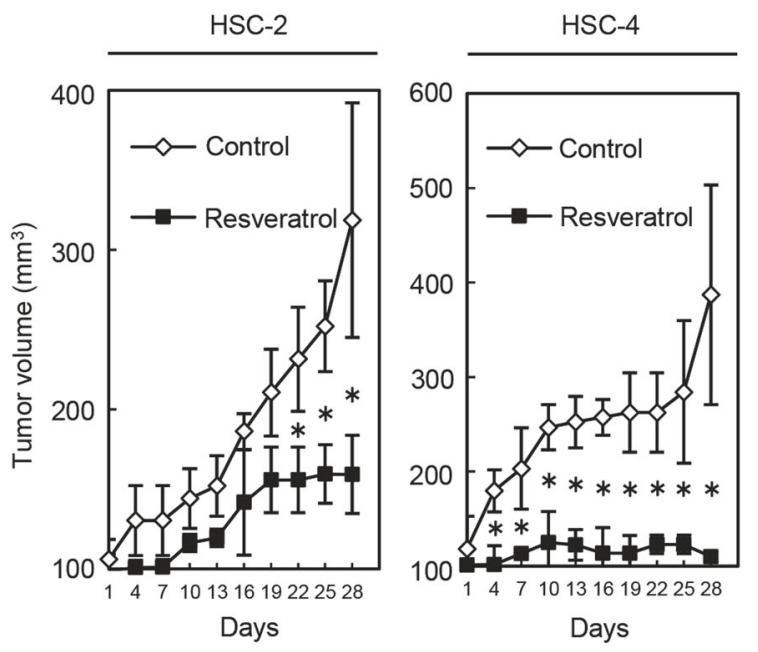

b
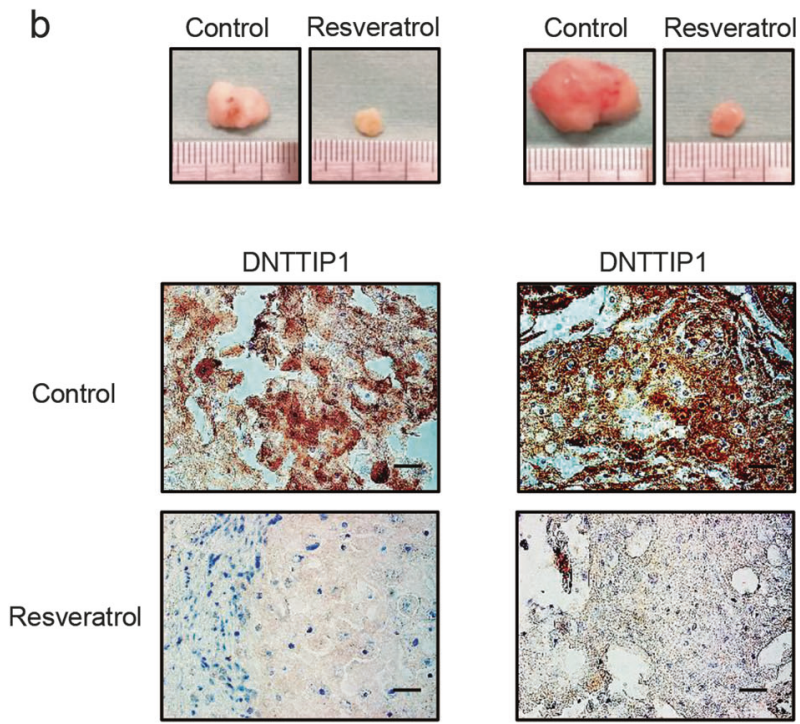

Fig. 7 Resveratrol inhibits tumoral growth in vivo. a Female nude mice (6-weeks old; BALB/cA-nu [nu/nu]) with tumor xenografts were injected daily intraperitoneally with vehicle (DMSO) or resveratrol (2 $\mathrm{mg}$ of resveratrol in $50 \mu \mathrm{l}$ of DMSO) for 28 days $(n=3)$. Tumoral growth in the resveratrol-treated group is inhibited significantly $(* P<$ 0.05) compared with the control group. b IHC of the xenografted tumors shows decreased immunostaining for DNTTIP1 in the resveratrol-treated group

Acknowledgements We thank Ms. Lynda C. Charters for editing this manuscript. The authors received no financial support.

\section{Compliance with ethical standards}

Conflict of interest The authors declare that they have no conflict of interest.

\section{References}

1. Yamashita N, Shimazaki N, Ibe $S$, et al. Terminal deoxynucleotidyltransferase directly interacts with a novel nuclear protein that is homologous to p65. Genes Cells. 2001;6:641-52. 
2. Fleming-Waddell JN, Wilson LM, Olbricht GR, et al. Analysis of gene expression during the onset of muscle hypertrophy in callipyge lambs. Anim Genet. 2007;38:28-36.

3. Fujita K, Shimazaki N, Ohta Y, et al. Terminal deoxynucleotidyltransferase forms a ternary complex with a novel chromatin remodeling protein with $82 \mathrm{kDa}$ and core histone. Genes Cells. 2003;8:559-71.

4. Motea EA, Berdis AJ. Terminal deoxynucleotidyl transferase: the story of a misguided DNA polymerase. Biochim Biophys Acta. 2010;1804:1151-66.

5. Yamano Y, Uzawa K, Shinozuka K, et al. Hyaluronan-mediated motility: a target in oral squamous cell carcinoma. Int $\mathrm{J}$ Oncol. 2008;32:1001-9.

6. Pande V. Understanding the complexity of epigenetic target space. J Med Chem. 2016;59:1299-307.

7. Weichert W. HDAC expression and clinical prognosis in human malignancies. Cancer Lett. 2009;280:168-76.

8. Juan LJ, Shia WJ, Chen MH, et al. Histone deacetylases specifically down-regulate p53-dependent gene activation. J Biol Chem. 2000;275:20436-43.

9. Bantscheff M, Hopf C, Savitski MM, et al. Chemoproteomics profiling of HDAC inhibitors reveals selective targeting of HDAC complexes. Nat Biotechnol. 2011;29:255-65.

10. Itoh T, Fairall L, Muskett FW, et al. Structural and functional characterization of a cell cycle associated HDAC1/2 complex reveals the structural basis for complex assembly and nucleosome targeting. Nucl Acids Res. 2015;43:2033-44.

11. Tanzawa H, Uzawa K, Kasamatsu A, et al. Targeting gene therapies enhance sensitivity to chemo- and radiotherapy of human oral squamous cell carcinoma. Oral Sci Int. 2015; 12:43-52.

12. Shiiba Masashi, Ishige Shunsaku, Saito Yasuhiro, et al. Downregulated expression of family with sequence similarity 3 , member B (FAM3B), in oral squamous cell carcinoma. Oral Sci Int. 2012;9:9-16.

13. Kasamatsu A, Uzawa K, Usukura K, et al. Loss of heterozygosity in oral cancer. Oral Sci Int. 2011;8:37-43.

14. Endo Y, Uzawa K, Mochida Y, et al. Sarcoendoplasmic reticulum $\mathrm{Ca}(2+)$ ATPase type 2 downregulated in human oral squamous cell carcinoma. Int J Cancer. 2004;231:225-31.

15. Kasamatsu A, Uzawa K, Nakashima D, et al. Galectin- 9 as a regulator of cellular adhesion in human oral squamous cell carcinoma cell lines. Int J Mol Med. 2005;16:269-73.

16. Miyamoto I, Kasamatsu A, Yamatoji M, et al. Kinesin family member 14 in human oral cancer: a potential biomarker for tumoral growth. Biochem Biophys Rep. 2015;3:26-31.

17. Koide Nao, Kasamatsu Atsushi, Endo-Sakamoto Yosuke, et al. Evidence for critical role of lymphocyte cytosolic protein 1 in oral cancer. Sci Rep. 2017;7:43379.

18. Unozawa M, Kasamatsu A, Higo M, et al. Cavin-2 in oral cancer: a potential predictor for tumor progression. Mol Carcinog. 2016;55:1037-47.

19. Shiiba M, Saito K, Fushimi K, et al. Lipocalin-2 is associated with radioresistance in oral cancer and lung cancer cells. Int $\mathrm{J}$ Oncol. 2013;42:1197-204.

20. Saito T, et al. Semaphorin7A promotion of tumoral growth and metastasis in human oral cancer by regulation of $\mathrm{g} 1$ cell cycle and matrix metalloproteases: possible contribution to tumoral angiogenesis. PLoS ONE. 2015;10:e0137923.

21. Kimura Y, et al. ARNT2 regulates tumoral growth in oral squamous cell carcinoma. J Cancer. 2016;7:702-10.

22. Uzawa $K$, et al. Long-term culture of human odontoma-derived cells with a Rho kinase inhibitor. Exp Cell Res. 2016;347:232-40.

23. Shimizu T, Kasamatsu A, Yamamoto A, et al. Annexin A10 in human oral cancer: biomarker for tumoral growth via G1/S transition by targeting MAPK signaling pathways. PLoS ONE. 2012;7:1-9.

24. Iyoda M, Kasamatsu A, Ishigami T, et al. Epithelial cell transforming sequence 2 in human oral cancer. PLoS ONE. 2010;5; e14082.

25. Baba T, Sakamoto Y, Kasamatsu A, et al. Persephin: a potential key component in human oral cancer progression through the RET receptor tyrosine kinase-mitogen-activated protein kinase signaling pathway. Mol Carcinog. 2013;617:1-8.

26. Shimizu F, Shiiba M, Ogawara K, et al. Overexpression of LIM and $\mathrm{SH} 3$ protein 1 leading to accelerated $\mathrm{G} 2 / \mathrm{M}$ phase transition contributes to enhanced tumourigenesis in oral cancer. PLoS ONE. 2013;8:1-9.

27. Yamatoji M, Kasamatsu A, Kouzu Y, et al. Dermatopontin: a potential predictor for metastasis of human oral cancer. Int $\mathrm{J}$ Cancer. 2012;130:2903-11.

28. Minakawa $\mathrm{Y}$, Kasamatsu A, Koike $\mathrm{H}$, et al. Kinesin family member 4A: a potential predictor for progression of human oral cancer. PLoS ONE. 2013;8:1-8.

29. Lombardi DP, Geradts J, Foley JF, et al. Loss of KAI1 expression in the progression of colorectal cancer. Cancer Res. 1999;59:5724-31.

30. Shimada K, Uzawa K, Kato M, et al. Aberrant expression of $\mathrm{RAB} 1 \mathrm{~A}$ in human tongue cancer. $\mathrm{Br} \mathrm{J}$ Cancer. 2005;92:1915-21.

31. Kouzu Y, Uzawa K, Koike H, et al. Overexpression of stathmin in oral squamous-cell carcinoma: correlation with tumour progression and poor prognosis. Br J Cancer. 2006;94:717-23.

32. Ferrero M, Ferragud J, Orlando L, et al. Phosphorylation of AIB1 at mitosis is regulated by CDK1/CYCLIN B. PLoS ONE. 2011;6: e28602.

33. Díaz-Rodríguez E, Álvarez-Fernández S, Chen X, Paiva B, et al. Deficient spindle assembly checkpoint in multiple myeloma. PLoS ONE. 2011;6:e27583.

34. Kai L, Samuel SK, Levenson AS. Resveratrol enhances p53 acetylation and apoptosis in prostate cancer by inhibiting MTA1/ NuRD complex. Int J Cancer. 2010;126:1538-48.

35. Dhar S, Kumar A, Li K, et al. Biochim Biophys Acta. Resveratrol Regul PTEN/Akt Pathw Inhib MTA1/HDAC Unit NuRD Complex Prostate Cancer. 2015;1853:265-75.

36. Sheth S, Jajoo S, Kaur T, et al. Resveratrol reduces prostate cancer growth and metastasis by inhibiting the Akt/MicroRNA-21 pathway. PLoS ONE. 2012;7:e51655.

37. Tsai TY, Chen TC, Wang IJ, et al. The effect of resveratrol on protecting corneal epithelial cells from cytotoxicity caused by moxifloxacin and benzalkonium chloride. Invest Ophthalmol Vis Sci. 2015;56:1575-84.

38. Watson PJ, Fairall L, Schwabe JW, Targeting Class I. Histone deacetylases in a "complex" environment. Millard CJ. Trends Pharmacol Sci. 2017;38:363-77.

39. Perisanidis, C, Perisanidis B, Wrba F, et al. Evaluation of immunohistochemical expression of p53, p21, p27, cyclin D1, and Ki67 in oral and oropharyngeal squamous cell carcinoma. J. Oral Pathol. Med. 2012;41:40-6.

40. Musgrove EA, Lee CS, Buckley MF, et al. Cyclin D1 induction in breast cancer cells shortens G1 and is sufficient for cells arrested in G1 to complete the cell cycle. Proc Natl Acad Sci USA. 1994;91:8022-6.

41. Tam SW, Theodoras AM, Shay JW, et al. Differential expression and regulation of cyclin D1 protein in normal and tumor human cells: association with Cdk4 is required for cyclin D1 function in G1 progression. Oncogene. 1994;9:2663-74.

42. Aggarwal BB, Bhardwaj A, Aggarwal RS, et al. Role of resveratrol in prevention and therapy of cancer: preclinical and clinical studies. Anticancer Res. 2004;24:2783-840. 
43. Jang M, Cai L, Udeani GO, et al. Cancer chemopreventive activity of resveratrol, a natural product derived from grapes. Science. 1997;275:218-20.

44. Kuwajerwala N, Cifuentes E, Gautam S, et al. Resveratrol induces prostate cancer cell entry into s phase and inhibits DNA synthesis. Cancer Res. 2002;62:2488-92.

45. Pozo-Guisado E, Lorenzo-Benayas MJ, Fernandez-Salguero PM. Resveratrol modulates the phosphoinositide 3-kinase pathway through an estrogen receptor alpha-dependent mechanism: relevance in cell proliferation. Int J Cancer. 2004;109:167-73.

46. Brakenhielm E, Cao R, Cao Y. Suppression of angiogenesis, tumor growth, and wound healing by resveratrol, a natural compound in red wine and grapes. FASEB J. 2001;15:1798-800.

47. Chung S,Yao H,Caito S,Rahman I, et al. Regulation of SIRT1 in cellular functions: role of polyphenols. Arch Biochem Biophys. 2010;501:79-90.

48. Park LK, Friso S, Choi SW. Nutritional influences on epigenetics and age-related disease. Proc Nutr Soc. 2012;71:75-83.
49. Dayangaç-Erden D, Bora G, Ayhan P, et al. Histone deacetylase inhibition activity and molecular docking of (e)-resveratrol: its therapeuticpotential in spinal muscular atrophy. Chem Biol Drug Des. 2009;73:355-64.

50. Venturelli S, Berger A, Böcker A, et al. Resveratrol as a PanHDAC inhibitor alters the acetylation status of jistone proteins in human-derived hepatoblastoma cells. PLoS ONE. 2013;8:e73097

51. Dayangaç-Erden D, Bora G, Ayhan P, et al. Histone deacetylase inhibition activity and molecular docking of (e)-resveratrol: its therapeutic potential in spinal muscular atrophy. Chem Biol Drug Des. 2009;73:355-64.

52. Zhang S, Cao HJ, Davis FB, et al. Oestrogen inhibits resveratrolinduced post-translational modification of p53 and apoptosis in breast cancer cells. Br J Cancer. 2004;91:178-85.

53. Narayanan BA, Narayanan NK, Re GG, et al. Differential expression of genes induced by resveratrol in LNCaP cells: P53mediated molecular targets. Int J Cancer. 2003;104:204-12. 